J. H. J. Walton), pp. 181-191. Montreal: Heol Publications.

O. O. FamuYtwa, Associate Professor, Department of Psychiatry, College of Medicine, University of Lagos, PMB12003, Lagos, Nigeria

\section{Psychotherapy experience for trainees}

Sir: Debate within the College has suggested that compulsory psychotherapy experience for psychiatric trainees would lead to an erosion of opportunity for research experience. Published research is widely seen as a prerequisite for progression through the training grades (Duffet, 1994), yet concern has been raised as to whether it is feasible for trainees to pursue meaningful research before moving into the specialist registrar/senior registrar grade (Timini, 1995). It may be that trainees involved in research are doing so at the expense of psychotherapy training or vice versa. An alternative, but perhaps less charitable, viewpoint is that while some trainees are keen to do both research and psychotherapy, others consistently manage to avoid doing both.

To investigate this further we conducted a questionnaire survey of psychiatric trainees at registrar level training with the South Thames (West) training scheme based at St George's Hospital, Tooting, South London. All registrars training in psychiatry in the Region in 19951996 received a two-part questionnaire. The first part focused on how many psychotherapy cases the trainee had treated, subdivided into modalities including cognitive-behavioural and individual psychodynamic psychotherapy. The second part of the questionnaire asked about the number and type of research projects the trainee was involved in.

Fifty-four questionnaires were received from 56 trainees giving a response rate of $96 \%$. Direct involvement in research activity was claimed by $79 \%$ of the sample; $93 \%$ had treated one or more cognitive-behavioural cases, while $85 \%$ had treated one or more individual psychodynamic cases. Kendall's tau correlation coefficients showed no association between number of research projects and number of individual psychodynamic cases treated $(\tau=0.092$, $P=0.404)$. There was, however, a significant correlation between number of research projects and number of behavioural cases treated ( $\tau=0.336, P=0.003$ ).

These findings do not support the idea that trainees who make time for treating psychotherapy cases do so at the expense of research involvement. Trainees treating cognitive-behavioural psychotherapy cases appear able to combine activity in both and should continue to be encouraged to do so.

DufFET, R. (1994) Publication by junior doctors: why do they do it? Psychiatric Bulletin. 18, 553-554.

Timimi, S. (1995) Trainee psychiatrists' theoretical vacuum (letter). Psychiatric Bulletin. 19. 707.

JOHN GING and LYNNE M. DRUMMOND, Section of Psychotherapy. St George's Hospital Medical School, Jenner Wing, Cranmer Terrace, London SW17 ORE

\section{Evidence-based medicine}

Sir: I read with interest Schmidt et al's editorial on evidence-based medicine (EBM) (Psychiatric Bulletin, December 1996, 20, 705-707). I would like to add a few pertinent details from Sacket $e t$ al (1996) (also listed in Schmidt et als article).

Sacket (Director of NHS Research \& Development Centre for EBM, Oxford, UK) and co-workers use a comprehensive definition of EBM: ". . . the conscientious, explicit and judicious use of current best evidence in making decisions about the care of individual patients". Its practice "... means integrating individual clinical expertise with the best avallable external evidence from systematic research". If there is no available evidence that fulfils gold standards, then "... . we follow the trail to the next best external evidence and work from there".

Schmidt et al depict a scenario where insisting on the best option may augment a patient's resistance to treatment or affect the doctorpatient relationship. A clinical decision process must include the patient's relative preferences (i.e. utilities), or better still, the values that the patient assigns to such utilities. Only when a patient cannot do this might the clinician alone quantify these utilities. In either situation, the final decision may not necessarlly favour the option best supported by the external evidence. Thus, Sacket et al argue that external clinical evidence ". . . can never replace individual clinical expertise and it is this expertise that decides whether the external evidence applies to the individual patient at all, and, if so, how it should be integrated into a clinical decision"; that is, EBM strengthens but does not supplant clinical expertise.

Schmidt et als assumption that many will feel unable to appraise research articles critically is not a strong argument to dismiss EBM. For many it may take some practice to become proficient, but the same applies to the development of most other skills. 